\title{
Dispositivos tecnológicos: uso académico en estudiantes universitarios
}

Fecha de recepción: 2021-08-15 • Fecha de aceptación: 2021-10-15 • Fecha de publicación: 2021-12-01

Dora Marcela Lliguisupa Pástor ${ }^{1}$ Universidad Estatal de Bolívar, Ecuador dlliguisupa@ueb.edu.ec

https://orcid.org/0000-0001-5808-6490

María de los Ángeles Bonilla² Universidad Estatal de Bolívar, Ecuador mabonilla@ueb.edu.ec https://orcid.org/0000-0003-2051-4626

Jonathan Patricio Cárdenas Benavides ${ }^{3}$ Universidad Estatal de Bolívar, Ecuador jcardenas@ueb.edu.ec https://orcid.org/0000-0002-9760-602X

\section{Resumen}

El estudio tuvo como objetivo conocer brevemente la frecuencia de uso de dispositivos tecnológicos en las carreras de educación básica y educación inicial, según el género. La investigación fue descriptiva con un enfoque cualitativo y con un corte transversal, además, por medio de un muestreo no probabilístico por conveniencia, se seleccionó a 227 estudiantes universitarios y se aplicó un cuestionario para conocer la frecuencia del uso de dispositivos tecnológicos según la carrera. Respecto al uso de dispositivos digitales entre las carreras de educación básica (EB) y educación inicial (EI), se pudo evidenciar que hay mayor frecuencia en la respuesta de "la mayoría de las veces" en EB en hombres (26), seguido del mismo grupo en mujeres (34), ante la misma respuesta. Se concluyó que la tecnología está transformando la educación, cambiando la forma, el momento y el lugar dónde aprenden los estudiantes, en tal sentido, se determinó que el uso de dispositivos 
es considerablemente frecuente, siendo el teléfono móvil el dispositivo más usado, y como consecuencia, puede marcar una gran diferencia en la educación.

Palabras clave: dispositivos tecnológicos, tecnología, frecuencia de uso, estudiantes universitarios

\begin{abstract}
The objective of the study was to briefly know the frequency of use of technological devices in basic education and early childhood education majors, according to gender. The research was descriptive with a qualitative approach and with a cross-sectional cut, in addition, by means of a non-probabilistic sampling by convenience, 227 university students were selected and a questionnaire was applied to know the frequency of the use of technological devices according to the career. Regarding the use of digital devices between basic education (BE) and early childhood education (IE), it was found that there is a higher frequency of "most of the time" in BE in men (26), followed by the same group in women (34), for the same answer. It was concluded that technology is transforming education, changing the way, the time and the place where students learn, in this sense, it was determined that the use of devices is considerably frequent, being the cell phone the most used device, and as a consequence, it can make a big difference in education.
\end{abstract}

Keywords: technological devices, technology, frequency of use, university students 


\section{Introducción}

La convergencia de la comunicación y la informática para dispositivos tecnológicos de consumo está en un curso evolutivo para brindar interoperabilidad y aprovechar los servicios y funciones de todas y cada una de las industrias. El uso de computadoras portátiles, tabletas, teléfonos móviles y otros dispositivos, se ha convertido en un elemento permanente en el panorama actual. Un rápido progreso de los dispositivos tecnológicos en la sociedad temporal, plantea una cuestión de su impacto en los seres humanos que se discute ampliamente en sociología y filosofía. Si bien existe un crecimiento de la literatura sobre los criterios y componentes del uso de dispositivos tecnológicos, poco se ha hecho para conocer la frecuencia de uso que subyacen a su desarrollo.

Desde que los docentes han tratado de enseñar a los estudiantes, existen teorías que guían cómo esos docentes ven el proceso de aprendizaje. Estas teorías del aprendizaje abarcan creencias sobre la naturaleza del conocimiento y cómo aprende una persona (Medina et al., 2019). Las teorías del aprendizaje han existido durante milenios, e incluso en el mundo moderno, existe una gran diversidad en la forma en que los científicos, psicólogos y educadores ven el aprendizaje (Torrenteras, 2012). Algunas de las principales teorías del aprendizaje que dan forma a las conversaciones modernas, en torno a la integración de la tecnología, incluyen el conductismo, cognitivismo, constructivismo, construccionismo y conectivismo.

El mundo se está convirtiendo en una red inteligente de personas, dispositivos, contenido y servicios habilitados digitalmente. Los nuevos dispositivos pueden aprender y adaptarse a las necesidades de las personas en función de situaciones específicas, ubicación y contexto de uso. Además, ser conscientes del contexto para que puedan predecir las necesidades de los usuarios en diferentes contextos, siempre un paso por delante de ellos y eventualmente haciendo superfluas las interfaces de usuario La brecha tecnológica, en gran parte, es direccionada al acceso o uso de la tecnología digital, pero también en poder integrar la tecnología digital en prácticas sociales significativas y obtener beneficios (Gómez et al., 2018). La generación joven debe comprender y tomar decisiones informadas sobre cómo utilizar las tecnologías en la vida cotidiana de manera significativa. Además, la brecha tecnológica también afecta al diseño y desarrollo de dicha tecnología, es importante que la generación joven adopte una postura crítica y proactiva hacia los dispositivos tecnológicos.

Ciertamente, el uso de la tecnología por parte de los jóvenes para comunicarse entre sí, no es nada nuevo; sin embargo, lo que ha cambiado es la forma que toma la comunicación, por ejemplo, las tecnologías basadas en texto (Barquero \& Calderón, 2016). Hoy en día, debido al auge de la tecnología, se ha direccionado hacia un enfoque más digital para mantener y establecer relaciones tanto en un contexto social, como educativo, que es tanto fomentado, como mediada por un dispositivo digital.

La revolución digital se ha involucrado profundamente en la vida diaria, evidente en la ubicuidad de los dispositivos tecnológicos y la perfecta integración de la tecnología en tareas comunes (Manríquez, 2018). El uso de computadoras y otros dispositivos se encuentra en su nivel más alto hasta la fecha y se espera que continúe aumentando a medida que la tecnología se vuelva 
más accesible, particularmente para los usuarios de los países en desarrollo (Marcano, 2014). Un mayor acceso y demanda de tecnología ha presentado oportunidades y desafíos únicos para muchas industrias, algunas de las cuales han prosperado al digitalizar eficazmente sus operaciones y servicios (por ejemplo, finanzas, medios), y otras que han luchado por mantenerse al día con el ritmo de la innovación tecnológica (por ejemplo, educación, atención médica). Por lo descrito, el objetivo de la investigación fue conocer brevemente la frecuencia de uso de dispositivos tecnológicos según las carreras de educación básica y educación inicial, según el género.

\subsection{Dispositivos tecnológicos}

Los dispositivos tecnológicos se refieren a una amplia gama de tecnologías que almacenan y transmiten información en forma digital y podrían estar basadas en hardware/dispositivos (como computadoras, teléfonos móviles y otros dispositivos móviles, cámaras digitales, video y audio, jugadores, consolas de juegos, etc.); o basado en software (aplicaciones web, blogs, wikis, sitios de redes sociales, juegos de computadora, sitios de chat, etc.). El término dispositivos digitales, incluye computadoras móviles -o portátiles-, dispositivos móviles (también conocidos como dispositivos de mano) y herramientas de comunicación inalámbrica (Ruano et al., 2016)los sujetos encuentran diversos medios para trasmitir información , construir aprendizajes , incrementar la productividad y establecer contacto con otros. Estas herramientas, se han popularizado por permitir la posibilidad de expresar y compartir con el resto del mundo todo tipo de emociones , experiencias y acontecimientos, concibiendo la comunicación y la interacción desde un plano psicológico y social. Este trabajo aborda cambios psicológicos generados en los estilos de comunicación y de interacción en un entorno educativo, evidenciando la presencia de cuatro tipologías de usuarios consumidores tecnológicos (nativos, inmigrantes, reflexivos y escépticos. La tecnología de comunicación móvil se define como dispositivos y servicios que apoyaron la conectividad social mediada, mientras el usuario está en movimiento físico (Romero \& Rivera, 2019).

Por otra parte, las redes sociales móviles se definen como softwares, aplicaciones o servicios a los que se accede a través de dispositivos móviles que permiten a los usuarios conectarse con otras personas y compartir información, noticias y contenido (Castro, 2012)haciendo especial hincapié en aquellas que favorecen la inclusión de las personas con necesidades educativas especiales. A modo de introducción, se resume la evolución de estas tecnologías, y a continuación, se desarrolla una nueva concepción del aprendizaje en línea y un modelo de innovación basado en el concepto de aprendizaje invisible y en los sistemas ubicuos de aprendizaje. Se define una plataforma de Aprendizaje digital, integrando una serie e-Servicios y Tecnologías de Apoyo, que es un ecosistema de aprendizaje en línea, interactivo, inteligente, inclusivo, accesible, usable, ubicuo, adaptativo, multimodal y diseñado para Todos. Este sistema actúa como elemento facilitador de la interacción entre el usuario y los objetos y actividades de aprendizaje, teniendo en cuenta que el público objetivo son las personas con diversidad y aquellas que nunca han utilizado las tecnologías digitales por miedo (tecnofobia. Las redes sociales móviles tienen diferentes tipos de servicios, como (micro) blogs (por ejemplo, Twitter), sitios de redes sociales (por ejemplo, Facebook, Tuenti), wikis (por ejemplo, Wikipedia), servicios para compartir videos o fotos (por ejemplo, YouTube y Flickr), servicios de recomendación y servicios para compartir la ubicación. 
Los dispositivos son las únicas tecnologías que pueden estar en todas partes y en todo momento, dando acceso inmediato e individual a una amplia gama de diferentes medios de comunicación. El teléfono móvil, es una tecnología a la que accede un grupo más amplio de usuarios que les proporciona acceso a canales de comunicación, tecnologías de video y audio, acceso a Internet y mensajes de texto. Originalmente pensados para la comunicación basada en voz, los teléfonos móviles a menudo incluyen capacidades de mensajería de texto que facilitan nuevas formas de interacciones sociales.

\subsection{Dispositivos tecnológicos}

Integrar la tecnología en la enseñanza y el aprendizaje no es un desafío nuevo, desde la década de 1900, los administradores y el profesorado se han enfrentado a cómo utilizar de manera eficaz las innovaciones técnicas, como las grabaciones de audio y video, el correo electrónico y las teleconferencias para aumentar o reemplazar los métodos tradicionales de impartición de instrucción (Schindler et al., 2017). Sin embargo, en las últimas dos décadas, este desafío ha sido mucho más difícil debido al gran volumen de nuevas tecnologías en el mercado. Un ejemplo claro de esto es que en el lapso de 7 años (de 2008 a 2015), el número de aplicaciones activas en la App Store de Apple aumentó de 5000 a 1,75 millones (Schindler et al., 2017).

Durante la última década, la tecnología digital, en particular los dispositivos como tabletas, lectores electrónicos, computadoras portátiles compactas y teléfonos inteligentes, han hecho que el conocimiento sea accesible y el aprendizaje sea portátil (Basantes et al., 2017). La era digital, presenta desafíos y transformaciones en los sistemas educativos (instituciones, docentes, estudiantes, familia), pero simultáneamente ofrece nuevas oportunidades para la enseñanza, el aprendizaje y la pedagogía (Capilla, 2016).

Los dispositivos tecnológicos nuevos y emergentes ofrecen el potencial de transformar las aulas, en entornos de aprendizaje más atractivos, colaborativos y productivos en los que el aprendizaje se puede personalizar según las necesidades, intereses y preferencias de aprendizaje específicos de los estudiantes. Otras tecnologías colaborativas en línea tienen el potencial de redefinir la forma en que los educadores enseñan, así como el papel que desempeñan los educadores, desde ser la única fuente de información, hasta ser un guía, facilitador y entrenador en el proceso de aprendizaje (Bonilla Jurado et al., 2018)nuevos productos, menores costos.

En el mundo desarrollado, los estudiantes que han crecido en la era digital llegan a las instituciones educativas con habilidades y experiencias en el uso de una variedad de tecnologías digitales (Mora et al., 2018). Las tecnologías emergentes requieren nuevas habilidades y estrategias por parte de los estudiantes, como búsqueda en la biblioteca en línea, habilidades informáticas básicas, habilidades de indagación y pensamiento crítico, análisis correcto y uso eficiente de los recursos y herramientas digitales en línea.

Además, las universidades están cada vez más preocupadas por desarrollar la alfabetización digital y las habilidades del siglo XXI entre todos los estudiantes, por ejemplo, la capacidad para la creatividad, resolución colaborativa de problemas, investigación, alfabetización en información digital y ciudadanía (Gallardo, 2014). 
Hoy en día, los académicos ven más allá de este enfoque de considerar la alfabetización digital como una mera habilidad para usar un paquete de software y también enfatizan la importancia de saber usar los dispositivos digitales en un contexto social (García, 2017). En consecuencia, implica tanto la experiencia como las habilidades en el uso mecánico, así como el conocimiento y las habilidades sobre el uso de estos dispositivos con fines de comunicación, redes sociales, educación, investigación, empresas comerciales, entre otros aspectos.

\subsection{Teorías de aprendizaje}

En la actualidad, y sobre todo en el contexto educativo, la modalidad de enseñanza y la forma de aprender se encuentra en un constante cambio, considerando que el Internet, dispositivos digitales, herramientas y recursos evolucionan de forma acelerada (Uribe, 2017). Las teorías de aprendizaje parten desde un ámbito tradicional y en sus inicios no se consideró al ámbito de la tecnología en la educación. Aspectos notorios, debido a lo trascendental del cambio evolutivo en la educación, sustentando que, en la actualidad, existen millones de personas que se encuentran en un constante uso de las tecnologías, lo cual, en consideración al aprendizaje, se construye conocimientos aportando al desarrollo evolutivo del conocimiento con una contribución e interacción colaborativa.

El conocimiento procedente del uso de dispositivos digitales, es un contexto más ampliado del propio conocimiento, el cual está relacionado a las experiencias generadas en un entorno. En tal sentido, las teorías de aprendizaje como la cognitivista y la conductista, hasta la actualidad, han efectuado aportes realmente significativos, considerando que, modificaron y constituyeron la concepción del aprendizaje, formulando modelos y diversos enfoques educativos. Ahora, estas teorías en sus inicios solo se direccionaron al individuo como único ente protagonista de su propio conocimiento. No obstante, no fue considerado el entorno de aprendizaje, así como la tecnología y su respectiva interacción. La teoría constructivista incorpora y analiza el entorno de aprendizaje y la teoría más recientemente centrada en la era digital es el conectivismo, teoría que involucra los aspectos y elementos que no se consideraron en teorías ya tradicionales.

\subsubsection{Conductismo}

Se popularizó a mediados del siglo XX, cuando los psicólogos estudiaron patrones de comportamiento y sistemas de respuesta en humanos y otros animales (Leiva, 2005). El conductismo trata el aprendizaje como una respuesta a un estímulo (Suárez, 2013). Es decir, los humanos y animales están entrenados para responder de determinadas formas a determinados estímulos, como salivar cuando suena la campana de una cena o repetir un hecho memorizado para recibir alguna recompensa externa. Entonces, la enseñanza y el aprendizaje es un proceso de acondicionamiento de los estudiantes para que reaccionen adecuadamente a los estímulos y la tecnología puede ayudar a facilitar esta capacitación, al proporcionar incentivos al aprendizaje como juegos u otras, recompensas, o al proporcionar sistemas para desarrollar eficientemente el condicionamiento de estímulo-respuesta, como las prácticas de taladrar y matar. 


\subsubsection{Cognitivismo}

Surgió como una alternativa al conductismo, en parte porque el conductismo trataba los procesos del cerebro como una caja negra imperceptible, en la que comprender cómo funcionaba el cerebro no se consideraba importante para ayudar a las personas a aprender (Zapata, 2015). El cognitivismo, por lo tanto, se ocupa de las funciones cerebrales y de cómo se procesa, almacena, recupera y aplica la información (Ramírez, 2009). Al tratar a los humanos como máquinas pensantes, en lugar de animales a entrenar, la investigación en cognitivismo para la enseñanza y el aprendizaje se centró en ayudar a las personas a desarrollar estrategias eficientes de enseñanza y estudio que permitirían a sus cerebros hacer un uso significativo de la información presentada. A través de esta lente, la tecnología puede ayudar a proporcionar información y recursos de estudio que ayuden al cerebro a almacenar y recuperar información de manera eficiente.

\subsubsection{Constructivismo}

Sin embargo, tanto el conductismo como el cognitivismo tendían a tratar el aprendizaje por igual para todos los seres humanos, a pesar de su edad, cultura o experiencias personales (Ortiz, 2015). Reconociendo que estos factores pueden influir en cómo ocurre el aprendizaje, el constructivismo surgió como un medio para comprender cómo los factores individuales y sociales pueden influir en el proceso de aprendizaje de diferentes grupos de personas e individuos (Muñoz, 2015). El constructivismo sostiene que el aprendizaje lo construyen los alumnos sobre la base de experiencias, actitudes y creencias previas. Esto significa que para que se produzca el aprendizaje, las nuevas experiencias de aprendizaje deben tener en cuenta estos factores humanos y ayudar al individuo a asimilar los nuevos conocimientos a sus construcciones de conocimientos existentes. Por lo tanto, si está enseñando a los estudiantes sobre fracciones, debe enseñarles usando un lenguaje que comprendan y conecten su aprendizaje con experiencias en sus propias vidas, que tendrán significado para ellos.

Creyendo que el conocimiento se construye en la mente, algunos llevaron el constructivismo a la etapa de un proceso pedagógico y lo llamaron construccionismo (Rintaningrum, 2008). Desde el punto de vista construccionista, la forma más efectiva de enseñar de manera constructivista es hacer que los estudiantes construyan artefactos en el mundo exterior, que apoyen y reflejen su construcción interna del conocimiento (Bhattacharjee, 2015). Por ejemplo, si un estudiante necesita aprender conceptos básicos de ingeniería, con el fin de construir los modelos mentales internos necesarios para comprender la ingeniería, estos deben construir modelos externos, que pueden tomar la forma de un puente o catapulta, por ejemplo. La tecnología puede respaldar los enfoques construccionistas de la enseñanza y el aprendizaje al capacitar a los estudiantes y maestros para crear y construir modelos externos que reflejen modelos mentales internos con recursos y posibilidades que no están disponibles en el mundo real. Al usar una simulación, por ejemplo, los estudiantes pueden construir cualquier estructura o máquina, sin la necesidad de materiales costosos. 


\subsubsection{Conectivismo}

Incluso con estas teorías en competencia, algunos todavía creían que las experiencias y procesos de aprendizaje, tal como existen en el mundo real, no estaban completamente representados, y esto se ha vuelto especialmente obvio ahora que vivimos en una sociedad que está fuertemente interconectada y conectada a través de medios electrónicos y sociales (medios de comunicación) (Gutiérrez, 2012). Todos los puntos de vista tradicionales sobre el aprendizaje habían colocado el conocimiento y el aprendizaje directamente en la mente o el cuerpo del estudiante, pero las tecnologías modernas, en particular, llevan a considerar si toda la memoria, el procesamiento de información y otros aspectos del aprendizaje tradicionalmente atribuidos a la mente, podrían no serlo también distribuido con dispositivos externos (Morrás, 2014). El conectivismo sostiene que el proceso y los objetivos del aprendizaje en un mundo altamente interconectado y conectado, es diferente al aprendizaje en el mundo predigital, porque los estudiantes ahora están conectados de manera persistente a fuentes de información y otros recursos a través de sus dispositivos electrónicos, como teléfonos inteligentes o computadoras portátiles.

Desde la perspectiva conectivista, el aprendizaje no tiene por qué estar aislado de la mente, pero convertirse en un ciudadano instruido y capaz en una sociedad digital requiere que los alumnos se conecten entre sí, de tal manera que puedan hacer uso de la red como una extensión de su propia mente y cuerpo. Por lo tanto, desde una perspectiva conectivista, el objetivo de la educación es conectar de manera más completa y eficiente a los estudiantes entre sí y con los recursos de información de una manera que sea persistente y en la que los alumnos puedan hacer un uso continuo de la red para resolver problemas.

\subsection{Uso de los dispositivos tecnológicos en estudiantes universitarios}

Los dispositivos tecnológicos (DT) son herramientas que facilitan el desarrollo de la sociedad por medio de la información, en lo que el conocimiento sufre diversas metamorfosis, lo cual da espacio para múltiples verdades, conocimiento, estilos de interacción e identidad. La construcción individual y colectiva entre las experiencias y discursos que se dan entre la realidad virtual y su efecto en la realidad física y viceversa (Martínez, 2011).

En sustento a las teorías de aprendizaje, y en consideración al uso y la aplicación del docente, existen muchos dispositivos tecnológicos que los estudiantes universitarios utilizan, ya sea para mejorar la instrucción o para apoyar su aprendizaje. Los dispositivos tecnológicos pueden extender el aprendizaje de maneras poderosas. Los estudiantes y docentes pueden dar diversos usos a los dispositivos, tales como:

- Acceso a material de fuente primaria actualizada.

- Métodos de recopilación / registro de datos.

- Formas de colaborar con estudiantes, profesores y expertos a nivel mundial.

- Oportunidades para expresar comprensión a través de multimedia.

- Aprendizaje relevante y evaluación auténtica. 
- Capacitación para publicar y presentar sus nuevos conocimientos.

- Desarrollar la capacidad de los estudiantes para la investigación

- Permitir la presentación de información en formas atractivas

- Ofrece acceso a recursos de aprendizaje

Considerando a los nativos tecnológicos y escépticos, se entiende que el uso de estas herramientas es diferente según el escenario, en el primero, su uso es de más alta frecuencia para entretenimiento, comunicación, interacción, aprendizaje, trabajo y necesidades; el escéptico, en cambio, tiene una frecuencia de uso muy baja o nula en estos DT para realizar actividades específicas, en muchas ocasiones relacionadas con correos institucionales o cosas obligatorias (Ruano et al., 2016)los sujetos encuentran diversos medios para trasmitir información , construir aprendizajes, incrementar la productividad y establecer contacto con otros. Estas herramientas, se han popularizado por permitir la posibilidad de expresar y compartir con el resto del mundo todo tipo de emociones, experiencias y acontecimientos, concibiendo la comunicación y la interacción desde un plano psicológico y social . Este trabajo aborda cambios psicológicos generados en los estilos de comunicación y de interacción en un entorno educativo, evidenciando la presencia de cuatro tipologías de usuarios consumidores tecnológicos (nativos, inmigrantes, reflexivos y escépticos. Entonces se puede observar que hay abstención (escéptico) y satisfacción (nativo).

El tiempo al día dedicado a DT en su mayoría, es al dispositivo de comunicación móvil (celular), con un promedio de tres horas aproximadamente, seguido del televisor y el computador con dos horas y media respectivamente. También se entiende que a mayor edad, mayor frecuencia en tiempo eran usados los DI, especialmente el celular (Franco, 2013). En jóvenes de Guayaquil perteneciente en la provincia de Guayas en Ecuador se entiende que las actividades en el tiempo libre, son por medio de DT en el uso más frecuente para chatear $(50,16 \%)$, redes sociales $(55,41 \%)$ y navegar en Internet $(35,08 \%)$, analizando por cada pregunta al respecto. Al cuestionar por medio de DT se usa para acceder al Internet, se conoció que el $63 \%$ usa el teléfono celular.

Para conocer la frecuencia en tiempo que se usa los DT, se evidenció que 131 personas lo usan más de 2 horas, seguido de 1 a 2 horas (90) de un total muestral de 305 usuarios que participaron en la investigación. Como se puede ver, hay un alto nivel de uso de los DT con mayor frecuencia en el uso del celular en tiempo y con finalidades diferentes. Lo cual, confirma un análisis del uso de DT de la juventud del Guayas en el que se ve por años el porcentaje de personas que tienen celular activado y se puede percibir el aumento de esto entre los años 2012 al 2016 (Del Pozo et al., 2018).

Esto puede tener efectos sobre las personas y puede marcar de forma significativa en una investigación realizada sobre el uso y abuso de las tecnologías y su relación con algunas variables de personalidad, estilos de crianza, consumo de alcohol y autopercepción. En esto se conoció que el uso del teléfono móvil por la frecuencia vinculado al sexo tiene relación significativa, en lo cual también se vio que mayor cantidad de chicas afirmaron querer dejar este DT, pero no lo consiguieron. Además, se notó por parte de las mujeres que hacen uso abusivo del este artefacto, es decir, que en esta investigación el género femenino tuvo mayor consciencia del uso de ese 
artefacto, la forma en que se imposibilitaba dejarlo y que si afecta su vida cotidiana. Al cuestionar si "costaría mucho vivir sin el móvil", los varones (131) refirieron que les constaría menos estar sin él. Se constato en general que las mujeres, por cantidad o frecuencia de respuesta, tienen menos control sobre el uso del celular, deteriorado relaciones familiares y afectando su propia salud (Garrote, 2013).

De esta forma, se puede ver que la frecuencia del uso de DT pueden causar una gran afectación en las personas. Sin embargo, también son herramientas que permiten alcanzar información que anteriormente sería imposible. De la misma forma da partida a conocer y crear nuevas cosas innovadoras en la actualidad, por lo cual depende la forma y la razón de su uso frecuente, que decidirá sobre su efecto. A continuación, se expresará un caso práctico sobre la frecuencia de los DT, con el objetivo de conocer brevemente el uso de DT según las carreras de educación básica y educación inicial, según el género.

\section{Metodología}

El estudio es descriptivo, ya que busca detallar el "qué", de la investigación, tomando en cuenta las características del artículo. Se trabajó bajo un enfoque cualitativo debido a que se recolectaron y se analizaron los datos obtenidos para poder sustentar. Se obtuvo la información en un tiempo específico, por lo cual se llevó un corte transversal. Esto con un muestreo no probabilístico por conveniencia, donde se selecciona una muestra basada en criterios de inclusión (Hernández Sampieri \& Mendoza Torres, 2018). Estos criterios de inclusión contienen que sean estudiantes universitarios pertenecientes a la carrera de educación básica y educación inicial. Restringiendo a todas las personas que no cumplían con estas características.

Ante una muestra de 227 estudiantes universitarios se aplicó un cuestionario para conocer la frecuencia del uso de dispositivos tecnológicos, según la carrera. La aplicación se realizó por medio de Google drive, lo cual permitió obtener lo datos para este análisis de caso práctico. Los indicadores que contenían esta evaluación son descriptores de carrera según género y frecuencia de uso de dispositivos. De esta forma, conocer por último la frecuencia de género, carrera y uso de dispositivos, permitiendo describir y establecer relación ante las situaciones expresadas.

\section{Resultados}

A continuación se evidencia la interpretación de los resultados obtenidos 
Figura 1.

Datos demográficos

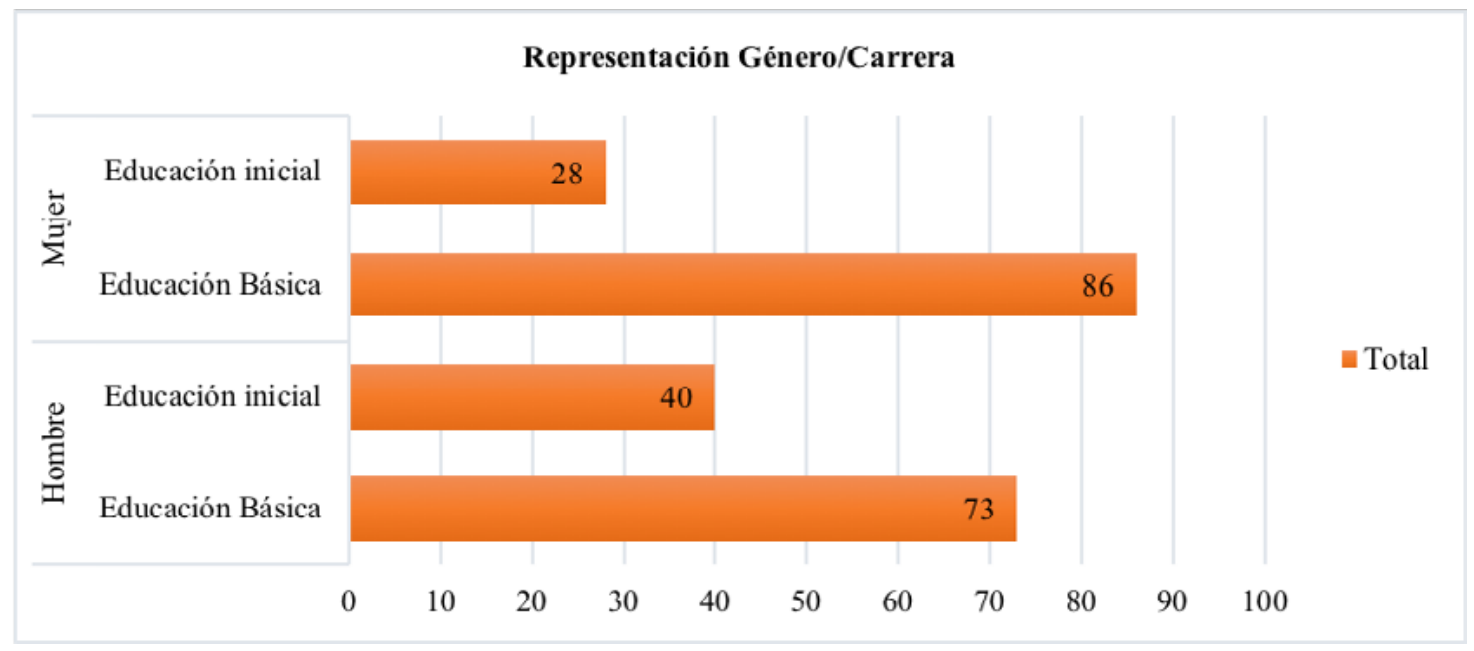

En el análisis de estudiantes según genero/carrera, se puede percibir mayor frecuencia $(F)$ de mujeres en la carrera educación básica (86), como se observa en la Figura 1. Seguido de la cantidad en $\mathrm{F}$ en hombres en educación básica. A diferencia de esto, la $\mathrm{F}$ inferior fue en educación inicial en mujeres. Esto refiere que hay más mayor población a fin de la carrera de educación básica en ambos géneros, los cuales harán uso del material para solventar las necesidades educativas.

Figura 2.

Frecuencia de uso de dispositivos digitales

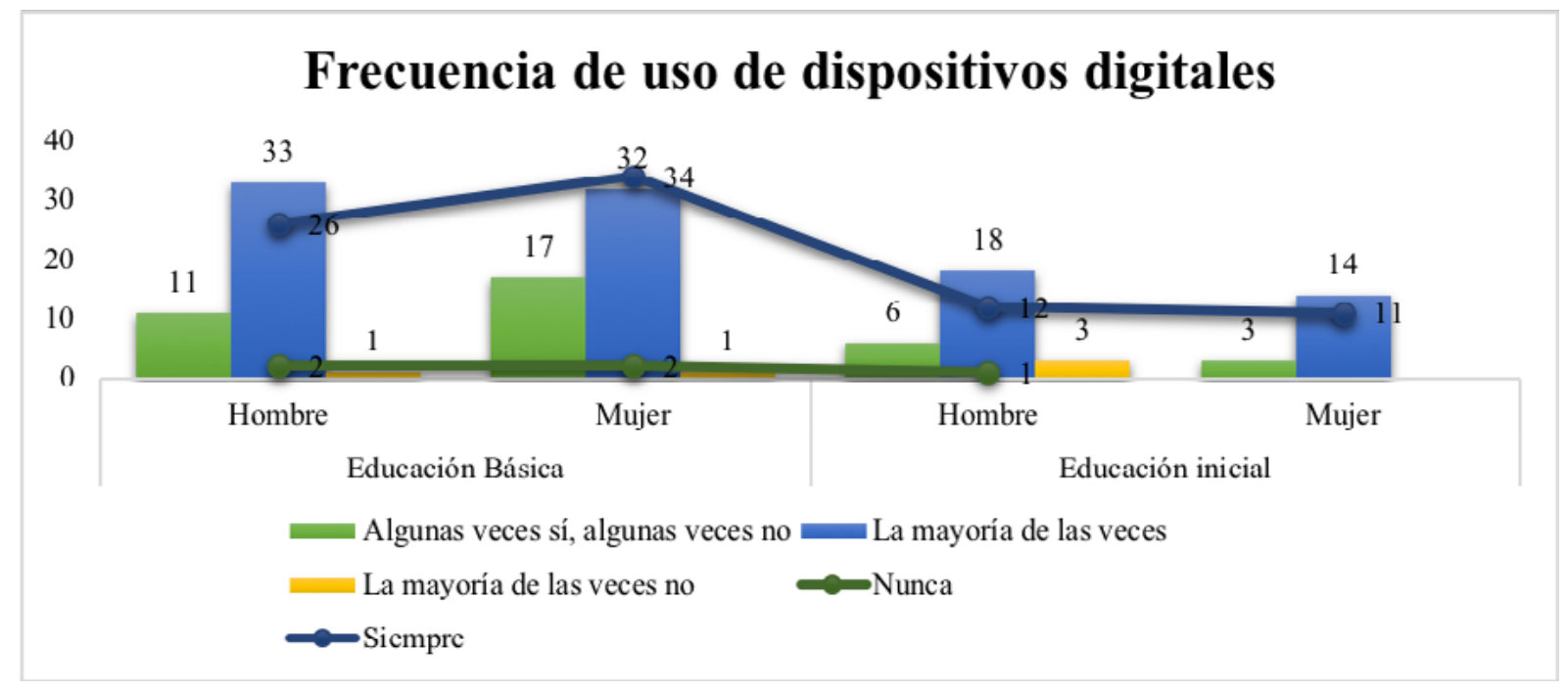

Respecto al uso de dispositivos digitales entre las carreras de educación básica (EB) y educación inicial (EI) tomando en cuenta hombres y mujeres de cada grupo. Se puede ver que hay mayor frecuencia en la respuesta de "la mayoría de las veces" en EB en hombres (26) seguido del mismo 
grupo en mujeres (34) ante la misma respuesta (ver Figura 2). Fenómeno que se repite en El en la misma respuesta entre los mismos géneros (18 hombres; 14 mujeres). A diferencia de esto, la respuesta "la mayoría de las veces no" es la menos puntuada en EB en ambos sexos, cosa que se repite en El. Esto significa que general hay un alto nivel de uso de este tipo de artefactos, lo cual con el objetivo de solventar las necesidades particulares de cada persona parte de esta investigación.

Figura 3.

Género, carrera y uso de dispositivos

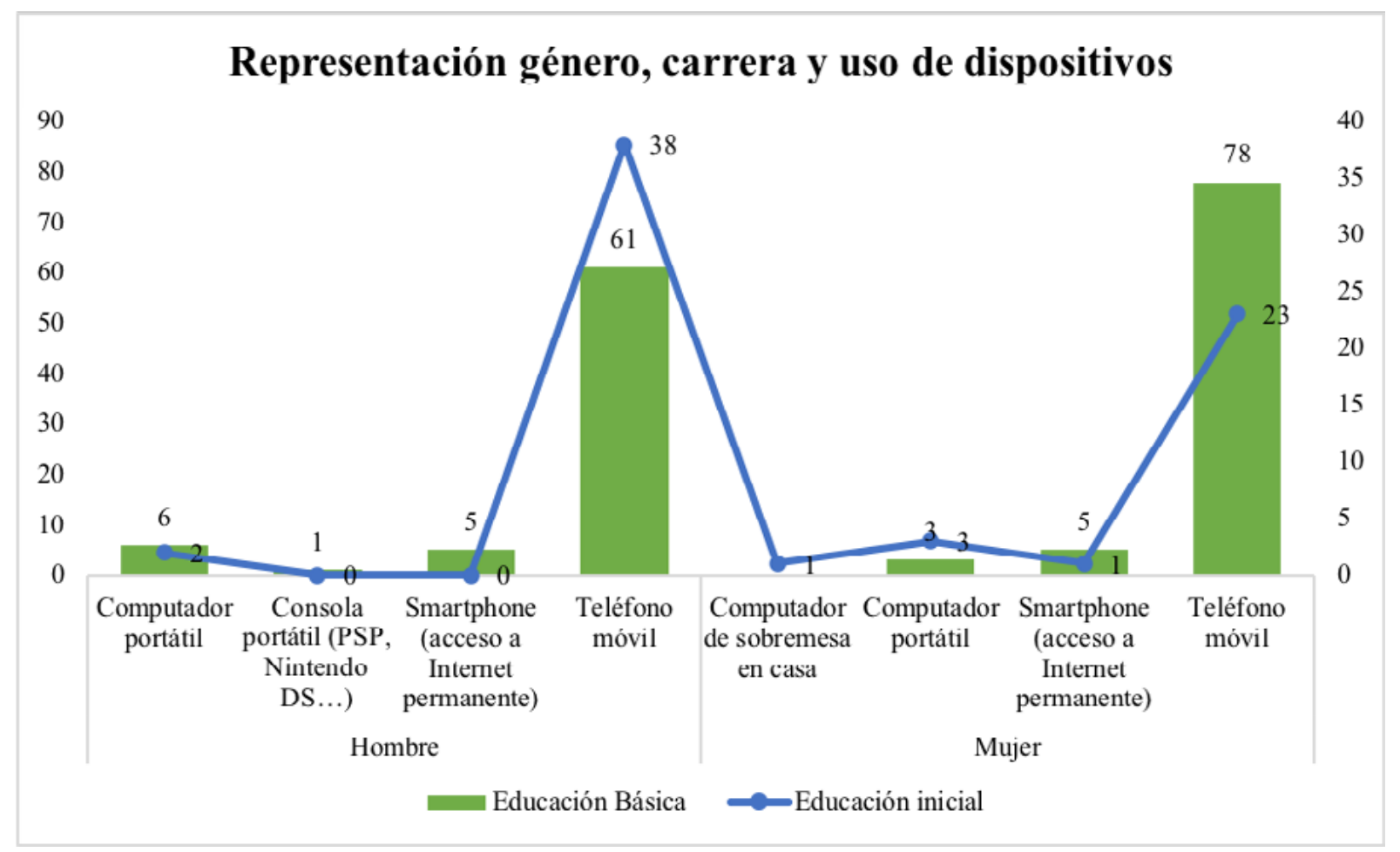

Al relacionar el género, carrera y uso de dispositivos, se puede percibir dos grandes puntos opuestos (Figura 3). Tanto en ambos géneros, como en las dos carreras, las personas usan el teléfono móvil con mayor frecuencia, las razones de esto pueden ser diversas, como comunicación, diversión, ocio, entre otros. Por otro lado, computador portátil, consola portátil y smartphone son lo que menos se usa por parte de los estudiantes que formaron parte de esta investigación. Esto puede significar que estas personas tienen mejor alcance hacia lo que necesitan individualmente por medio de sus teléfonos móviles.

Se pudo ver que, tanto en los grupos de hombre, como en mujeres, la mayor parte de estudiantes se encontraban en la carrera de educación básica. En los mismos que al ver la frecuencia de uso de dispositivos, la mayoría de veces la opción de mayor frecuencia. Lo cual refiere que tanto hombre como mujeres usan estos dispositivos gran parte de su tiempo, principalmente en educación básica. Al analizar género, carrera y uso de dispositivos, se conoció que, tanto en educación básica, como en educación inicial, hombre y mujeres usan más el teléfono móvil, esto 
puede ser por demandas individuales personales y también por exigencias educativas, haciendo de estos artefactos una herramienta de apoyo.

\section{Conclusiones}

A modo de conclusión, la tecnología está transformando la educación, cambiando la forma, el momento y el lugar dónde aprenden los estudiantes, se ha convertido en una parte integral de la sociedad, el uso de los dispositivos tecnológicos puede marcar una gran diferencia en la educación. Por lo tanto, se determinó que el uso de dispositivos es considerablemente frecuente, siendo el teléfono móvil el dispositivo más usado.

Cuando la tecnología está disponible y su funcionamiento es correcto, los estudiantes pueden acceder a la información más actualizada de forma más rápida y sencilla. El modelo tradicional de aprendizaje pasivo cada vez es menos vigente y en la actualidad, la tecnología transforma la experiencia de aprendizaje. Los dispositivos tecnológicos jamás dejan de avanzar, el costo del aprendizaje y desarrollo de la educación se ha reducido con la presencia de nueva tecnología, el resultado del aprendizaje se ha vuelto rentable, por lo que los dispositivos móviles juegan un papel importante para facilitar el aprendizaje.

El uso de estas tecnologías ha facilitado muchas metodologías educativas efectivas, como el aprendizaje autodirigido, independiente y colaborativo. Puede conectar a personas que, separadas por horario y ubicación, y puede brindar la oportunidad de recibir retroalimentación y evaluación inmediatas, haciendo que el aprendizaje parezca comparativamente más alcanzable de lo que sería sin retroalimentación instantánea. 


\section{Referencias}

Barquero, A., \& Calderón, F. (2016). Influencia de las nuevas tecnologías en el desarrollo adolescente y posibles desajustes. Revista Cúpula, 30(2), 11-25.

Basantes, A., Naranjo, M., Gallegos, M., \& Benítez, N. (2017). Los dispositivos móviles en el proceso de aprendizaje de la facultad de educación ciencia y tecnología de la universidad técnica del norte de Ecuador. Formación Universitaria, 10(2), 79-88. https://doi.org/10.4067/S0718-50062017000200009

Bhattacharjee, J. (2015). Constructivist approach to learning an effective approach of Teaching Learning. Research Journal of Interdisciplinary \& Multidisciplinary Studies (IRJIMS), 1(6), 65-747969.

Bonilla Jurado, D., Oña Sinchiguano, B., \& López Núñez, H. (2018). Medición de innovación tecnológica como eje central del crecimiento empresarial familiar del sector carrocero de la Provincia de Tungurahua. Revista Lasallista de Investigación, 15(2), 271-285. https://doi.org/10.22507/rli.v15n2a21

Capilla, R. (2016). Habilidades cognitivas y aprendizaje significativo de la adición y sustracción de fracciones comunes. Cuadernos de Investigación Educativa, 7(2), 49-62.

Castro, C. (2012). El futuro de las tecnologías digitales aplicadas al aprendizaje de personas con necesidades educativas especiales. Revista de Educación a Distancia, (32). https://revistas.um.es/red/article/ view/233051

Del Pozo, V., Vernimmen, G., \& Estrella, I. (2018). Análisis del uso de dispositivos tecnológicos en la juventud de la provincia del Guayas. En Uso del teléfono móvil, juventud y familia, 199-212. https://hdl.handle. $\underline{\text { net/11441/87877 }}$

Franco, A. (2013). El uso de la tecnología: determinación del tiempo que los jóvenes de entre 12 y 18 años dedican a los equipos tecnológicos. RIED Revista Iberoamericana de Educación a Distancia, 16(2), 107-125. https://doi.org/10.5944/ried.16.2.9908

Gallardo, E. (2014). An investigation of the social and academic uses of digital technology by University Students. [Tesis doctoral, Universitat Rovira I Virgil]. https://core.ac.uk/download/pdf/33346039.pdf

García, S. (2017). Alfabetización Digital. Razón y Palabra, 21(3_98), 66-81. https://revistarazonypalabra.org/ index.php/ryp/article/view/1043

Gómez, D., Alvarado, R., Martínez, M., \& Díaz, C. (2018). La brecha digital: una revisión conceptual y aportaciones metodológicas para su estudio de México. Entreciencias: Diálogos En La Sociedad Del Conocimiento, 6(16), 47-62. https://doi.org/10.22201/enesl.20078064e.2018.16.62611

Gutiérrez, L. (2012). Conectivismo como teoría de aprendizaje: conceptos, ideas, y posibles limitaciones. Revis- 
ta Educación y Tecnología, (1), 111-122.

Hernández Sampieri, R., \& Mendoza Torres, C. P. (2018). Metodología de la investigación las rutas cuantitativa, cualitativa y mixta. Editorial McGraw Hill.

Leiva, C. (2005). Conductismo, cognitivismo y aprendizaje. Tecnología En Marcha, 18(1), 4-8.

Manríquez, A. (2018). El uso de TIC en la comunicación con la ciudadanía: diagnóstico de portales web gobiernos locales en México. [Tesis doctoral, Universidad Complutense de Madrid]. Eprints UCM https://eprints. ucm.es/id/eprint/50692/1/T40750.pdf

Marcano, I. (2014). Apropiación de las tecnologías de información y comunicación en el ámbito educativo venezolano. Revista Educación, 39(1), 121. https://doi.org/10.15517/revedu.v39i1.9836

Martínez, J. (2011). Tecnoestrés: Ansiedad y adaptación a las nuevas tecnologías en la era digital. Ediciones Paidós.

Medina, J., Calla, G., \& Romero, P. (2019). Las teorías de aprendizaje y su evolución adecuada a la necesidad de la conectividad. Lex, 17(23), 377-387. https://doi.org/10.21503/lex.v17i23.1683

Mora, E., Bonilla, D., Nuñez, A., \& Sarmiento, C. (2018). Inadaptabilidad de los docentes al manejo de plataformas virtuales: Caso EDUCARECUADOR. Revista Conrado, 14(62), 39-43. http://scielo.sld.cu/scielo. php?pid=S1990-86442018000200006\&script=sci arttext\&tIng=en

Morrás, Á. (2014). Aportaciones del conectivismo como modelo pedagógico post-constructivista. Propuesta educativa, (42), 39-48.

Muñoz, M. (2015). La importancia del aprendizaje constructivista y la motivación en el aula de infantil. [Tesis de grado, Universidad Internacional de La Rioja]. Re-UNIR https://reunir.unir.net/handle/123456789/3313

Ortiz, D. (2015). El constructivismo como teoría y método de enseñanza. Sophia, (19), 93-110. https://doi. org/10.17163/soph.n19.2015.04

Garrote, G. (2013). Uso y abuso de tecnologías en adolescentes y su relación con algunas variables de personalidad, estilos de crianza, consumo de alcohol y autopercepción como estudiante. [Tesis doctoral, Universidad de Burgos]. Riubu. http://hdl.handle.net/10259/219

Ramírez, A. (2010). Importancia del paradigma cognitivo: Memoria y Procesamiento en Gagné. Retos y Redes, (2), 2-4.

Rintaningrum, R. (2008). Constructivist theory and a teaching and learning cycle in english. Jurnal Sosial Humaniora (JSH), 1(1). https://doi.org/10.12962/j24433527.v1i1.682 
Romero, L., \& Rivera, D. (2019). La comunicación en el escenario digital. Actualidad, retos y prospectivas. Editorial Pearson Educación.

Ruano, L., Congote, E., \& Torres, A. (2016). Dispositivos Tecnológicos: Comunicación e Interacción en un entorno Universitario. Atas - Investigação Qualitativa em Educação, 1, 48-57. https://proceedings.ciaiq.org/ index.php/ciaiq2016/article/view/588

Schindler, L., Burkholder, G., Morad, O., \& Marsh, C. (2017). Computer based technology and student engagement: a critical review of the literature. International Journal of Educational Technology in Higher Education, 14(1). https://doi.org/10.1186/s41239-017-0063-0

Suárez, R. (2013). Watson, Skinner y Algunas Disputas dentro del Conductismo. Revista Colombiana de Psicología, 22(2), 389-399.

Torrenteras, J. (2012). Las teorías de aprendizaje y la formación de herramientas técnicas. RED Revista de Educación a Distancia, (34). https://revistas.um.es/red/article/view/233531

Uribe, D. (2017). El aprendizaje en la era digital. Perspectivas desde las principales teorías. Aibi Revista de Investigación, Administración e Ingeniería, 5(2), 29-33. https://doi.org/10.15649/2346030x.439

Zapata, M. (2015). Teorías y modelos sobre el aprendizaje en entornos conectados y ubicuos. Eks, 16(1), 69-102. 
Copyright (c) 2021 Dora Marcela Lliguisupa Pástor, María de los Ángeles Bonilla y Jonathan Patricio Cárdenas Benavides

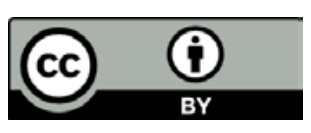

Este texto está protegido bajo una licencia internacional Creative Commons 4.0.

Usted es libre para Compartir-copiar y redistribuir el material en cualquier medio o formato - y Adaptar el documento - remezclar, transformar y crear a partir del material-para cualquier propósito, incluso para fines comerciales, siempre que cumpla las condiciones de Atribución. Usted debe dar crédito a la obra original de manera adecuada, proporcionar un enlace a la licencia, e indicar si se han realizado cambios. Puede hacerlo en cualquier forma razonable, pero no de forma tal que sugiera que tiene el apoyo del licenciante o lo recibe por el uso que hace de la obra.

\section{$\underline{\text { Resumen de licencia - Texto completo de la licencia }}$}

